\title{
Effects of ulinastatin on expression pattern of high mobility group box-1 protein and CD4+ CD25+ regulatory $T$ cells in rats with scald injury
}

\author{
HONGMING YANG ${ }^{*}$, CHAO HU ${ }^{*}$, YONGMING YAO ${ }^{l}$, JIAKE CHAI ${ }^{l}, M A I^{l}$, FENG YONGQIANG ${ }^{l}$, \\ CHU WANLI ${ }^{I}$
}

${ }^{1}$ First Hospital Affiliated to the Chinese PLA General Hospital, Beijing, PR China

${ }^{2}$ Beijing Luhe Hospital, Capital Medical University, Beijing, PR China

*Hongming Yang and Chao Hu contributed equally to this work.

\begin{abstract}
Aim of the study: To observe the effects of ulinastatin on Tlymphocyte immune function and the expression of high mobility group box-1 protein (HMGB1) in rats with scald injury.

Material and methods: A total of 96 Wistar rats were randomly divided into three groups (32 rats in each group): sham burn group $(S)$, burn group $(B)$, ulinastatin-treated group $(B U)$.

Sham-burned rats were immersed in distilled water at $37^{\circ} \mathrm{C}$ for 12 seconds and served as control, rats in the later two groups were inflicted with $30 \%$ TBSA full thickness burns at boiling water $\left(94^{\circ} \mathrm{C}\right)$ for 12 seconds, followed by resuscitation with Ringer's solution (i.p.). The treatment group $(B U)$ received ulinastatin and the control group was given the same amount of saline solution immediately following burn injury, wounds in rats were soaked in $1 \%$ povidone-iodine solution. Blood samples and spleens were collected at 1, 3, 5, 7 d after injury for further laboratory investigations. High mobility group box-1 protein expression was evaluated by western blotting, the presence of CD4+CD25+ regulatory $T$ cells were determined with flow cytometry, the plasma IL-4 and IFN- $\gamma$ levels were determined using ELISA.

Results: Compared with the S group $(0.22 \pm 0.05)$, during postburn days 1 to 7 , the level of HMGB1 in splenic tissue was significantly elevated in the B group $(p<0.01)$, reached the maximum at $3 d$ after injury $(0.66 \pm 0.10, p<0.01)$. After treated with ulinastatin, HMGB1 level was markedly decreased, reached the lowest value at $5 d(0.32 \pm 0.07, p<0.01)$. Compared with the sham burn group, percentage of CD4+CD25+ Treg cells in B group gradually increased after injury and peaked at $3 d$ after injury $(5.42 \% \pm 0.56 \%)$. After burn injury $1,5,7 \mathrm{~d}$, percentage of $C D 4+C D 25+$ Treg cells in $B U$ group was significantly lower than the B group $(p<0.01)$, reached the lowest at $5 d(2.23 \% \pm 0.21 \%)$. The serum level of IFN- $\gamma$ in B group was significantly higher than the $S$ group $(p<0.01)$, IL-4 was significantly higher than that of the $S$ group $(p<0.01)$. After injury for $1 \sim 7 d$, the content of IFN- $\gamma$ in $B U$ group were significantly higher than the B group $(p<0.05$ or $p<0.01)$, and IL-4 level was significantly lower than the burn group $(p<0.05$ or $p<0.01)$.

Conclusions: As an anti-inflammatory drug, ulinastatin effectively reduced the expression of HMGB1 in the spleen tissue of severely burned rats, which was associated with reduction in the level of regulatory T cells (Treg), and the functional polarization of splenic T cells levels and Th1/Th2 pattern changes. Taken together these data suggested that ulinastatin improved the immune function of rats with scald injury.
\end{abstract}

Key words: high mobility group box-1 protein, regulatory T cells, HMGB1, scald, ulinastatin, immunity.

(Centr Eur J Immunol 2013; 38 (1): 1-7)

Correspondence: Yang Hongming, 51 Fucheng Road, Haidian District, Beijing, People's Republic of China, tel. 8610-66848762, fax 8610-82636378, e-mail: hmyang126@126.com 


\section{Introduction}

CD4+ T cells have been subdivided into at least three functional Th subsets on the basis of their cytokine production, including Th1, Th2 and regulatory T (Treg) cells [1]. Especially, Treg cells, which are a subset of T lymphocytes that play critical role in immunological suppression and the termination of immune responses, and deficiency or dysfunction of these cells can lead to inflammatory and autoimmune diseases [2-5]. Tregs are broadly classified into two subsets: natural or adaptive (induced). Natural Tregs are CD4+CD25+ T-cells which develop and emigrate from the thymus to perform their key role in immune homeostasis. Based on CD25 expression, natural Tregs comprise 5-10\% of peripheral CD4+ T cells, and CD4+CD25+ Tregs have been found to suppress autoimmune $\mathrm{T}$ cell responses and reduce inflammation in a series of autoimmunity disorders [6]. CD4+CD25+ Tregs are important mediators of the suppression of $\mathrm{T}$ cell activation and the reduction in Th1 cytokine production found after injury [7].

High-mobility group box-1 (HMGB1) is a nuclear nonhistone DNA-binding protein that functions as a structural co-factor in gene transcription in somatic cells. Recent studies indicate that damaged or necrotic cells can release HMGB1 into the extracellular milieu, where it triggers inflammatory responses [8]. It was noted that serum levels of HMGB1 were increased in experimental burn injury models, and anti-HMGB1 antibodies decreased the severity of burn injury [9], demonstrating a vital role for HMGB1 in this pathophysiologic process. It was reported that the excessively released HMGB1 could stimulate CD4+CD25+ Tregs activity and trigger a shift of Th1 to Th2 with suppression of T-lymphocyte immune function after burn injury [10].

After severely burned, low levels of anti-inflammatory mediators and excessive expression of proinflammatory mediators leads to excessive inflammatory response, resulting in immune cell dysfunction and weak immune response [11]. The low-level of immune response is considered to be an important mechanism in the development of sepsis after burn injury (trauma). The purpose of the modern immunomodulation therapy is to prevent the body's inflammatory response caused by the immune intermediates into sepsis. Modulation of the Th1/Th2 balance has become a new paradigm for immunomodulatory therapy. Effective immunomodulation therapy is considered a fundamental breakthrough of sepsis treatment [12].

Ulinastatin, which is also known as urinary trypsin inhibitor, is a glycoprotein that was first detected in human blood and it functions as a broad-spectrum proteinase inhibitor. The inhibition of trypsin, $\alpha$-chymotrypsin, elastase, and various pancreatic enzymes by ulinastatin has been previously reported $[13,14]$. The administration of ulinastatin was reported to be effective in the treatment of acute pancreatitis, and a series of clinical trials have been carried out to confirm the safety and efficacy of the drug [15]. In clinical studies the most common side effects reported following intravenous administration of ulinastatin included skin rashes and angialgias at the injection site, abdominal symptoms, elevated liver enzymes, and other abnormalities in serum tests [16]. Anti-inflammatory effect of ulinastatin in septic rats has been confirmed [17], but the immune modulatory effects of ulinastatin remain to be elucidated. Its anti-inflammatory effects include suppressed production of the pro-inflammatory cytokines IL- 6 , IL- 8 , and TNF- $\alpha$, as well as reduced neutrophil infiltration [14]. The present study aimed to investigate the therapeutic effects of ulinastatin (intraperitoneally injected) on the expression of HMGB1 in rats with scald injury, the percentage of CD4+CD25+ Treg cells and the functional polarization of effector T cells. Our results provided the experimental basis for application of ulinastatin in treating immune dysfunction caused by excessive inflammation.

\section{Material and methods}

\section{Reagents and equipments}

Fluorescein isothiocyanate (FITC)-conjugated anti-rat antibody CD4, FITC-conjugated anti-mouse lgG2b (isotype control), phycoerythrin (PE)-conjugated anti-rat CD25 and PE-conjugated lgG2a (isotype control) were purchased from BD Pharmingen, USA. Recombinant HMGB1 proteins were purchased from Sigma (USA). Hemolysin was purchased from BD Pharmingen (USA). ELISA kits were purchased from R\&D Systems (USA). FACS Calibur flow cytometer was purchased from BD Pharmingen (USA). Ulinastatin was purchased from TianPu biochemical pharmaceutical Co., Ltd. (China, National Medical License Number H19990134).

\section{Animal model}

Ninety-six Wistar rats of SPF level, weighing 180-200 g, were purchased from the Beijing Vital River Lab Animal Co., Ltd, were housed in separate cages in a temperatureand humidity-controlled room with $12 \mathrm{~h}$ light and $12 \mathrm{~h}$ darkness, to acclimatize for at least 7 days before being used. All animals had free access to water but were fasted for 12 hours before the experiment. Wistar rats were randomly divided into three groups (32 rats in each group): sham burn group (S), burn group (B), ulinastatin-treated group (BU). All of these groups were further divided into four subgroups of eight rats each and they were killed on postburn days (PBD) 1, 3, 5, 7, respectively. Rats were anesthetized with intraperitoneal injection of pentobarbital sodium $(60 \mathrm{mg} / \mathrm{kg})$, and the animals' backs were shaved with $20 \%$ sodium sulfide. In the burn group (B) and ulinastatintreated group (BU) rats, a full skin thickness burn injury was produced in anesthetized rats by exposing $30 \%$ of total 
body surface area to $94^{\circ} \mathrm{C}$ water for $12 \mathrm{~s}$, and the animals' wounds were covered with $20 \mathrm{~g} / \mathrm{l}$ iodophors twice a day $[18,19]$. All the test animals were quickly dried after each exposure to avoid additional injury. The sham group rats were subjected to all of the procedures, except the temperature of the bath was at room temperature. Lactated Ringer solution $(40 \mathrm{ml} / \mathrm{kg}$ ) was administered intraperitoneally for delayed resuscitation $6 \mathrm{~h}$ after the injury and $4 \mathrm{ml}$ at 12, 24, 36, $48,60,72 \mathrm{~h}$ after the burn injury. Ulinastatin $\left(4 \times 10^{4} \mathrm{U} / \mathrm{kg}\right)$ was added to lactated Ringer solution in the ulinastatintreated groups. The wake animals after anesthesia were housed in separate cages, and were sacrificed on at designated time points, blood samples and spleens were collected, liquid nitrogen frozen and stored at $-80^{\circ} \mathrm{C}$ until further use. All experimental protocols were undertaken in accordance with the National Institutes of Health's Guide for the Care and Use of Laboratory Animals, with the approval of the scientific investigation board of the Burn Institute, Postgraduate Medical College, Beijing, China.

\section{Western blot analysis of HMGB1}

Expression of HMGB1 was analyzed by Western blotting as described previously. Spleen tissue lysate was prepared by homogenization in lysis buffer and the supernatant was collected after centrifugation at $14000 \mathrm{r} / \mathrm{min}$ for $30 \mathrm{~min}\left(4^{\circ} \mathrm{C}\right)$. The protein concentration was determined with BCA assay. For western blotting, samples $(50 \mu \mathrm{g})$ of total cell proteins were separated by SDS-PAGE, semi-dry transferred to polymembrane, after blocked by $5 \%$ defatted milk, the membrane was incubated with HMGB1 antibody (anti-rabbit, diluted $1: 1000$ ) overnight at $4^{\circ} \mathrm{C}$, washed and incubated with goat anti-rabbit antibody $(1: 2000)$, washed and visualized with an enhanced chemiluminescence substrate (Pierce, Rockford, Ill), exposed to X-ray film according to the manufacturer's instructions and scanned into a image scanner (LEICA Q-550IW; Wetzlar, Germany). Band intensities were quantified using National Institutes of Health Image 1.59 software and relative levels of HMGB 1 were normalized to that of the 30 pmol purified recombinant HMGB1 intensity (Sigma), which was run on each gel.

\section{Flow cytometric analysis}

Blood samples $(100 \mu \mathrm{l})$ were collected from abdominal aorta, treated with a hemolysis buffer $(17 \mathrm{mM}$ Tris- $\mathrm{HCl}$ and $140 \mathrm{mM} \mathrm{NH}_{4} \mathrm{Cl}, \mathrm{pH}$ 7.2) to remove red blood cells, stained with both FITC-conjugated anti-CD4 (5 $\mu \mathrm{g})$ and PE-conjugated anti-rat CD25 $(5 \mu \mathrm{g})$, incubated in the dark for 20 minutes $\left(4^{\circ} \mathrm{C}\right)$, appropriate PE- or FITC-conjugated irrelevant monoclonal antibody of the same Ig class was used as isotype control. Samples were washed twice with PBS, centrifuged at $1300 \mathrm{r} / \mathrm{min}$ and fixed in $1 \%$ paraformaldehyde. Isotype control antibody staining was used to set gates, numbers in gates reflect the percentage of pos- itive cells. Flow cytometry data were analyzed using the CellQuest software (Becton Dickinson Immunocytometry Systems, San Jose, CA).

\section{Interleukin 4, interferon $\gamma$ measurements by ELISA}

Serum IL-4, IFN- $\gamma$ levels were determined by ELISA, according to manufacturer's instructions. After enzyme substrate reaction was terminated, the absorbance of the yellow reaction mixture at $450 \mathrm{~nm}$ was then read using a microplate reader, a standard curve was produced with mean values of 6 duplicates in which absorbance values for blank tubes had been subtracted. The concentration of cytokines in samples could be calculated according to the standard curve.

\section{Statistical analysis}

Data were expressed as mean \pm standard deviation (SD) and analyzed with a one-way ANOVA. A Student-test was used to evaluate significant differences between groups. A $p$ value of 0.05 or less was considered to be statistically significant.

\section{Results}

\section{Animal mortality}

The total experimental animal number is 96 , and the number of animals that were included in the final grouping is 92 . Group $\mathrm{U}$ and $\mathrm{S}$ did not result in any mortality during the first $72 \mathrm{~h}$ of study. The animals in B group had an approximate $9.4 \%$ mortality in the same period. Most of the mortality occurred between 48 and $72 \mathrm{~h}$.

\section{HMGB1 expression pattern in spleen of all groups}

HMGB1 expression was analyzed by western blotting. Compared with the $S$ group $(0.22 \pm 0.05)$, during PBDs 1 to 7 , the relative expression level of HMGB1 in splenic tissue was significantly elevated in the B group $(p<0.01)$, peaking on PBD $3(0.66 \pm 0.10, p<0.01)$. After treated with ulinastatin, HMGB1 level was markedly decreased, reached the lowest value on PBD $5(0.32 \pm 0.07, p<0.01)$, while relative expression level of HMGB 1 in B group was 0.63 \pm 0.07 . There was statistical significance between $B$ groups and BU groups on PBD 1, 5, 7 (Fig. 1), while there was no obvious change between $\mathrm{B}$ groups and $\mathrm{BU}$ groups on PBD 3 (Fig. 1 and Fig. 2).

\section{CD4+CD25+ Treg expression}

A previous study in burnt mice showed that on days 1, 3 and 5 after injury, the highly purified population of splenic CD4+CD25+ T cells were predominately FoxP3+ Tregs, with approximately $70 \%$ of these cells staining positive for FoxP3 [10]. Therefore, we focused our current study on CD4+CD25+ T cells, which are predominately Tregs and not activated $\mathrm{T}$ cells. Flow cytometry analysis was per- 


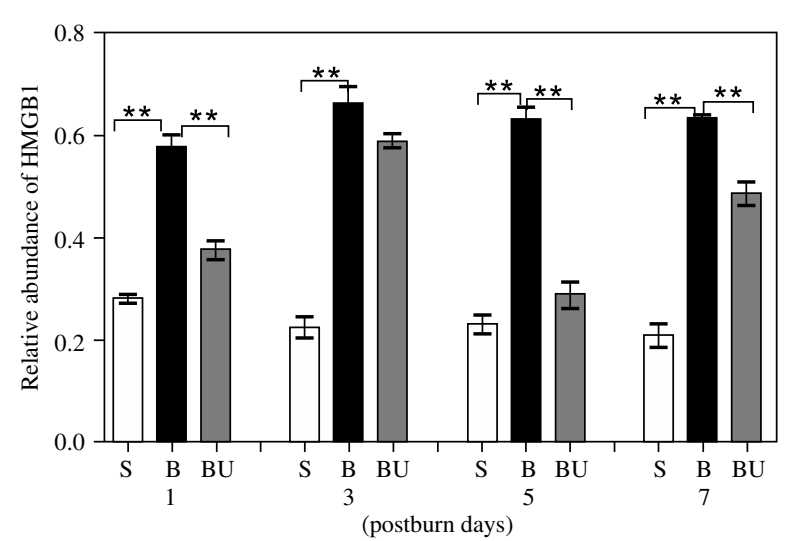

After subjected to scald injury, blood samples and spleen were collected at various time points. Compared with those in group S, HMGB1 expression in group B were significantly higher $(p<0.01)$, and peaked on day 3 ( $t$-value $=10.91$, $p<0.01$ ), about 3 fold of the $\mathrm{S}$ group. HMGB1 expression in group BU were all markedly lower than that in group B (all $p<0.01$ ), and it reached the lowest expression levels on day $5(t$-value $=9.61, p<0.01)$

Fig. 1. HMGB1 expression of all the groups at various time points

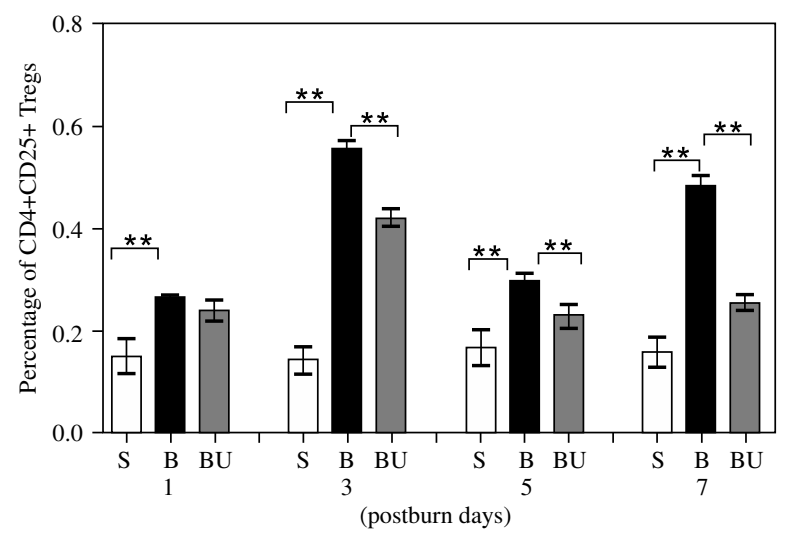

CD4+CD25+ Tregs of spleen or serum were analyzed by FACS at various time points. Compared with those in group BU, the percentages of CD4+CD25+ Treg gradually increased and peaked on day $3(5.42 \% \pm 0.56 \%)$. After treated with ulinastatin, the percentages of CD4+CD25+ Treg in group BU were obviously lower than that in group B on PBDs 3,5 , and $7(t$-value $=2.83,15.14,5.45$ and 22.26 , respectively) and it reached the lowest expression levels on day $5(2.23 \%$ $\pm 0.21 \%$ ). Each value represents the mean \pm SD of at least three measurements. The group B and group BU vs. the group $\mathrm{S},{ }^{\mathrm{a}} p<0.01$. Group $\mathrm{S}$ and the group $\mathrm{BU}$ vs. the group $\mathrm{B},{ }^{\mathrm{b}} p<0.01$

Fig. 3. Percentages of CD4+CD25+ Tregs

formed to evaluate the percentage of CD4+CD25+ Treg cells following treatment with ulinastatin or saline in burnt rats. It was observed that, compared with the $\mathrm{S}$ group, during PBDs 1 to 7, percentage of CD4+CD25+ Treg cells in $\mathrm{B}$ group gradually increased after injury $(t$-value $=2.83$, $15.14,5.45$ and 22.26; $p<0.01)$ and peaked at $3 \mathrm{~d}$ after injury $(5.42 \% \pm 0.56 \%)$. After injury $1,5,7 \mathrm{~d}$, percentage of CD4+CD25+ Treg cells in BU group was significantly lower than the burn group $(p<0.01)$, reached the lowest at $5 \mathrm{~d}(2.23 \% \pm 0.21 \%)$ (Fig. 3 and Fig. 4$)$.

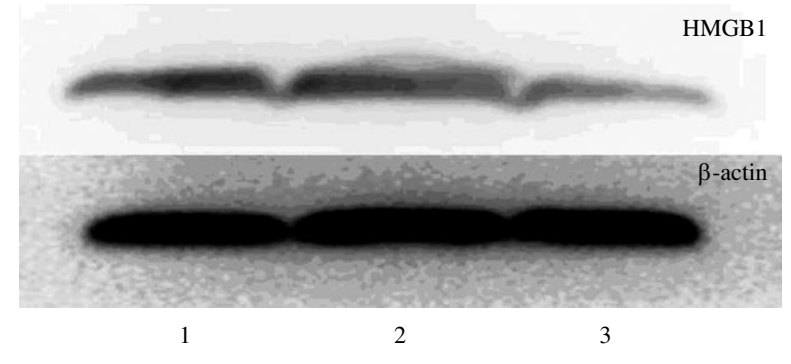

The spleen fractions were collected for western blot after different treatment. The lane 1 was the group S, lane 2 was group B and lane 3 was the group BU. $\beta$-actin was used as an internal control

Fig. 2. Western blot analysis of HMGB1 expression on PDB 3

\section{Interleukin 4 and interferon $\gamma$ expression}

The serum level of IFN- $\gamma$ in B group was significantly higher than the $\mathrm{S}$ group $(p<0.01)$, while IL-4 was significantly higher than that of the $S$ group $(p<0.01)$. After injury for $1 \sim 7 \mathrm{~d}$, the content of IFN- $\gamma$ in BU group were significantly higher than the $\mathrm{B}$ group $(p<0.05$ or $p<0.01)$, and IL-4 level was significantly lower than the B group $(p<0.05$ or $p<0.01)$ (Table 1$)$.

\section{Discussion}

Ulinastatin is a broad-spectrum protease inhibitor that is effective against a broad range of enzymes such as trypsin, hyaluronidase and other pancreatic enzymes. It has been used as a drug for patients with disseminated intravascular coagulation, shock, and pancreatitis in Japan [8-10]. Ulinastatin was observed to have the lysosomal membranestabilizing property and reduce the production of oxygen free radicals. Our results demonstrated that the percentage of regulatory CD4+CD25+ T lymphocytes (Treg) was elevated in severely burned rats, and peaked at $3 \mathrm{~d}$ after injury. Compared with the sham burn group, different expression levels of CD4+CD25+ Tregs in ulinastatin-treated group were observed, but significantly lower than those in burn group, indicating that the percentage of CD4+CD25+ Tregs was decreased in burnt rats after treated with ulinastatin.

CD4+CD25+ Tregs could efficiently induced type 2 T-cell polarization as demonstrated by the changes in IL-4/IFN- $\gamma$ ratio, which is an indicator of Th1/Th2 balance, indicating that lower the expression of CD4+CD25+ Tregs might efficiently induce type $1 \mathrm{~T}$-cell polarization, thus improving the immune function and reduce the risk of sepsis [20].

It was reported that the relative balance between IFN- $\gamma$ (Th1-type cytokine) and IL-4 (Th2-type cytokine) cytokines appeared crucial in the outcome of infections, IL-4 played critical role in modulating the Th1/Th2 cell polarization $[21,22]$. Serum IFN- $\gamma$ (Th1 cytokine) concentration of the burned mice decreased, while serum levels of IL-4 (Th2 cytokine) were elevated, thus promoted the Th2 polariza- 

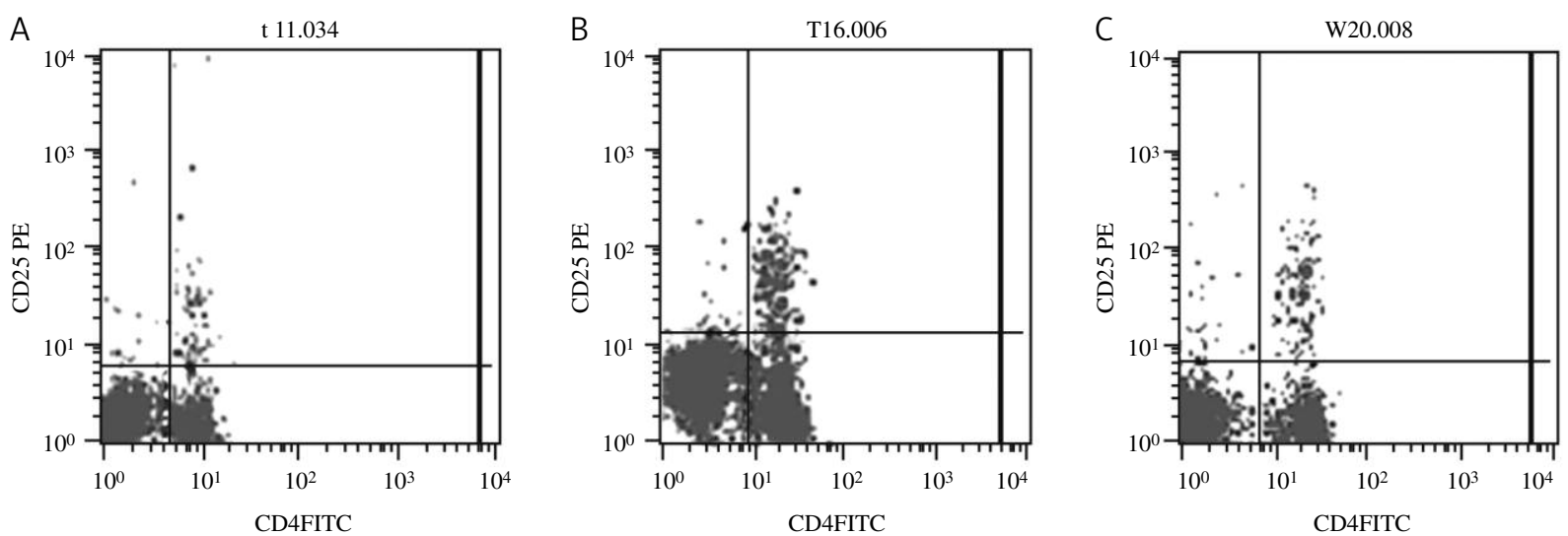

Uper left region: CD4(-)CD25(+), Lower left region: CD4(-)CD25(-), Uper right region: CD4(+)CD25(+), Lower right region: CD4(+)CD25(-). A: Expression of CD4(+)CD25(-) Tregs in S group at postburn 3d. B: Expression of CD4(+)CD25(+) Tregs in B group at postburn 3d. C: Expression of CD4(+)CD25(+) Tregs in $\mathrm{BU}$ group at postburn $3 \mathrm{~d}$.

A Group S; B Group B; C Group BU Note: top-left (TL): CD4(-)CD25(+); bottom-left (BL): CD4(-)CD25(-); top-right (TR): CD4(+)CD25(+); bottom-right (BR): $\mathrm{CD} 4(+) \mathrm{CD} 25(-)$

Fig. 4. FACS analysis of CD4+CD25+ Treg on PBD 3

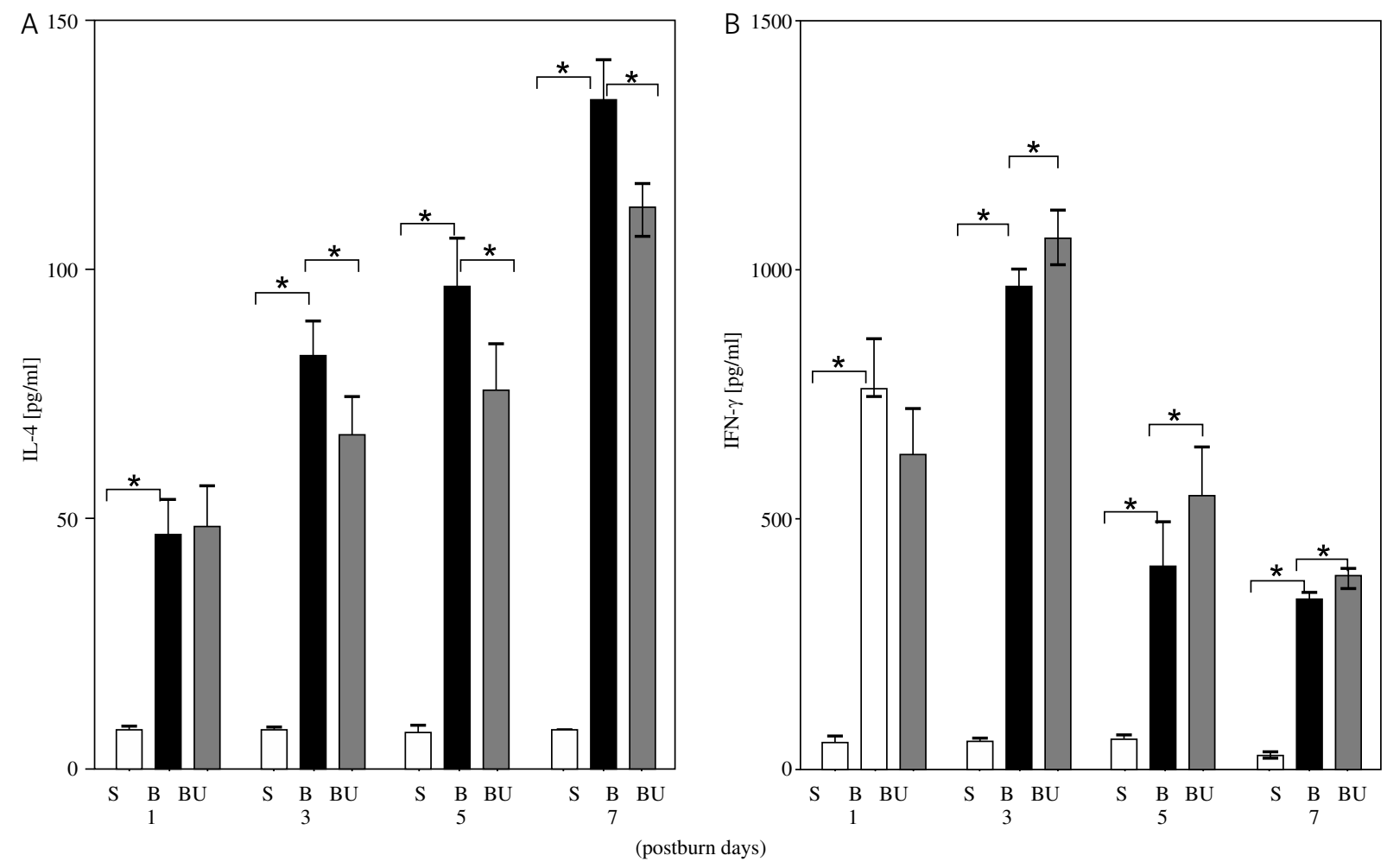

The group $\mathrm{B}$ and group $\mathrm{BU}$ vs. the group $\mathrm{S},{ }^{*} p<0.01$. The group $\mathrm{S}$ and the group $\mathrm{BU}$ vs. the group $\mathrm{B},{ }^{*} p<0.05$ and $* p<0.01$

Fig. 5. Enzyme-linked immunosorbent assay of the IL-4 and IFN- $\gamma$ levels in serum after injury $(n=8, \mathrm{pg} / \mathrm{ml})$

tion [23-25]. Serious injury induced a shift to the Th-2 phenotype with increased production of IL-4 rather than generalized Th suppression [26]. In present study, serum levels of IFN- $\gamma$ in the group B was significantly higher than the group $\mathrm{S}(p<0.01)$, while serum levels of IL-4 was sig- nificantly higher than the group $\mathrm{S}(p<0.01)$, indicating the percentage of Th2 polarized cells increased. After the BU group was treated with ulinastatin, the serum levels of IL-4 significantly decreased during PDBs 1 to 5, while serum levels of IFN- $\gamma$ significantly elevated during PDBs 
1 to 7 , demonstrating that ulinastatin efficiently induced Th1 cell polarization and the immune status was improved.

HMGB1 secreted as an important late-phase inflammatory mediators involved in the pathophysiological process of sepsis [27-29]. However, the mechanism of HMGB 1 synthesis and release after burn injury is still unclear, which may be associated with rapid release of various cytokines and endotoxin translocation under acute stress. Some studies showed that serum levels of HMGB1 were significantly elevated in patients with trauma or sepsis, which was associated with poor prognosis of patients, indicating that HMGB1 was closely related with occurrence of immune dysfunction and sepsis [30], providing new ideas and methods to combat immune dysfunction in sepsis. Studies have found that HMGB1 of severely burned rats may affect the inhibitory activity of regulatory $\mathrm{T}$ lymphocytes through RAGE (Receptor for Advanced Glycation End products), suppressed the proliferative response of $\mathrm{T}$ lymphocytes, played critical role the maturation process of CD4+CD25+ Treg in spleen of severely burned rats following delayed resuscitation, and mediated the subsets of $\mathrm{T}$ lymphocytes (T helper cells) differentiate into Th2 phenotype, then induced the inhibitory effects on immune function. To explore whether ulinastatin could inhibit the expression of HMGB1, as well as lead to decreased CD4+CD25+ Treg levels and Th1/Th2 pattern changes, we investigated the expression pattern of proinflammatory factor HMGB1 in burned rats. In this study confirmed that the levels of HMGB1 in spleen of burned rats were significantly elevated during PBDs 1 to $7 \mathrm{~d}$, peaking on PBD 3, and HMGB1 levels on PBD 7 were higher than in $5 \mathrm{~d}$. We speculated that severely burned rats were given only resuscitation and antiinflammatory treatment, lack of the necessary anti-infective therapy and nutritional support, the wound was still healing (wound debridement and healing stage), all these factors lead to the HMGB1 level increased again on PBD 7. After treated with ulinastatin, the expression levels of HMGB 1 were significantly decreased compared with burn group, suggesting that ulinastatin as anti-inflammatory drug effectively inhibited HMGB1 synthesis and release. In present study, the percentage of CD4+CD25+ Treg in T cells largely paralleled the HMGB1 expression pattern. Hence, we hypothesized that ulinastatin could efficiently suppress CD4+CD25+ Treg levels and inhibit Th2 cytokine profile (increased IFN- $\gamma$ and decreased IL-4 production), either directly or indirectly via the suppression of HMGB1 release.

In conclusion, our data support a putative role for ulinastatin-mediated suppression of HMGB1 expression in the reduction of CD4+CD25+ Treg levels and the pattern of Th1/Th2 polarization of splenic T cells. Further studies are needed to determine the exact mechanisms underlying this process, as well as its contribution to improved immune function of rats with scald injury. Moreover, in-depth studies in clinical trials into the pharmacological effects of ulinastatin should also be further elucidated.

\section{References}

1. Mosmann TR, Sad S (1996): The expanding universe of T-cell subsets: Th1, Th2 and more. Immunol Today 17: 138-146.

2. Zelenika D, Adams E, Mellor A (1998), et al. Rejection of $\mathrm{H}-\mathrm{Y}$ disparate skin grafts by monospecific CD4+ Th1 and Th2 cells: no requirement for CD8+ T cells or B cells. J Immunol 161: 1868-1874.

3. Saoudi A, Seddon B, Fowell D, Mason D (1996): The thymus contains a high frequency of cells that prevent autoimmune diabetes on transfer into prediabetic recipients. J Exp Med 184: 2393-2398.

4. Belkaid Y, Rouse BT (2005). Natural regulatory T cells in infectious disease. Nat Immunol 6: 353-360.

5. Mills KH (2004): Regulatory T cells: friend or foe in immunity to infection? Nat Rev Immunol 4: 841-855.

6. Mendez S, Reckling SK, Piccirillo CA, et al. (2004): Role for CD4 (+) CD25 (+) regulatory T cells in reactivation of persistent leishmaniasis and control of concomitant immunity. J Exp Med 200: 201-210.

7. Coutinho A, Caramalho I, Seixas E, Demengeot J (2005): Thymic commitment of regulatory $\mathrm{T}$ cells is a pathway of TCR-dependent selection that isolates repertoires undergoing positive or negative selection. Curr Top Microbiol Immunol 293: 43-71.

8. Scaffidi P, Misteli T, Bianchi ME (2002): Release of chromatin protein HMGB1 by necrotic cells triggers inflammation. Nature 418: 191-195.

9. Czura CJ, Tracey KJ (2003): Targeting high mobility group box 1 as a late-acting mediator of inflammation. Crit Care Med 31: S46-50.

10. Huang LF, Yao YM, Zhang LT, et al. (2009): The effect of high-mobility group box 1 protein on activity of regulatory $\mathrm{T}$ cells after scald injury in rats. Shock 31: 322-329.

11. Yao YM (2009): Current opinions on the potential mechanism, diagnosis and management for immune dysfunction of infections complications after trauma. Zhonghua Wai Ke Za Zhi 47: 37-39.

12. Holchkiss RS, Karl IE (2003): The pathophysiology and treatment of sepsis. N Engl J Med 348: 138-150.

13. Shikimi T, Hattori K, Takaori S (1993): Effects of heparin on the inhibitory activities of human urinary trypsin inhibitor (ulinastatin) on trypsin, chymotrypsin and leukocyte elastase. Jpn J Pharmacol 62: 115-118.

14. Umeadi C, Kandeel F, Al-Abdullah IH (2008): Ulinastatin is a novel protease inhibitor and neutral protease activator. Transplant Proc 40: 387-389.

15. Ikeda T, Ikeda K, Ohmi A, et al. (1994): The influence of ulinastatin on renal function during anesthesia for renal transplantation. Masui 43: 516-522.

16. Yamauchi Y, Izumi Y, Inoue M, et al. (2011): Safety of postoperative administration of human urinary trypsin inhibitor in lung cancer patients with idiopathic pulmonary fibrosis. PLoS One 6: e29053.

17. Cao YZ, Tu YY, Chen X, et al. (2012): Protective effect of Ulinastatin against murine models of sepsis: inhibition of TNF- $\alpha$ and IL-6 and augmentation of IL-10 and IL-13. Exp Toxicol Pathol 64: 543-547.

18. Chen XL, Xia ZF, Ben DF, et al. (2003): Role of p38 mitogen activated protein kinase in lung injury after burn trauma. Shock 19: 475-479.

19. Chen LW, Huang HL, Lee IT, et al. (2006): Thermal injuryinduced priming effect of neutrophil is TNF-alpha and P38 dependent. Shock 26: 69-76. 
20. Riley JL, June CH, Blazar BR (2009): Human T regulatory cells as therapeutic agents: take a billion or so of these and call me in the morning. Immunity 30: 656-665.

21. Antoniou KM, Ferdoutsis E, Bouros D (2003): Interferons and their application in the diseases of the lung. Chest 123: 209-216.

22. Yang X (2003): Role of cytokines in chlamydia trachomatis protective immunity and immune pathology. Curr Pharm Des 9: 67-73.

23. Matsuo R, Kobayashi M, Herndon DN, et al. (1996): Interleukin-12 protects scaldly injured mice from herpes simplex virus type 1 infection. J Leukoc Biol 59: 623-630.

24. Matsuo R, Herndon DN, Kobayashi M, et al. (1997): CD4CD8-TCR $\alpha / \beta+$ suppressor T cells demonstrated in mice 1 day after scald injury. J Trauma 42: 635-640.

25. Zedler S, Faist E, Ostermeier B, et al. (1997): Postburn constitutional changes in T-cell reactivity occur in $\mathrm{CD} 8+$ rather than CD4+ cells. J Trauma 42: 872-880.

26. O'Sullivan ST, Lederer JA, Horgan AF, et al. (1995): Major injury leads to predominance of the T-helper 2 lymphocyte phenotype and diminished interleukin-12 production associated with decreased resistance to infection. Ann Surg 224: 482-492.

27. Venet F, Chung CS, Kherouf H, et al. (2009): Increased circulating regulatory T cells $(\mathrm{CD} 4(+) \mathrm{CD} 25(+) \mathrm{CD} 127(-))$ contribute to lymphocyte anergy in septic shock patient. Intensive Care Med 35: 678-686.

28. Yao YM, Sheng ZY, Huang LF (2009): The effect of a novel cytokine, high mobility group box-1 protein, on the development of traumatic sepsis. Chin J Integr Med 15: 13-15.

29. Gardella S, Andrei C, Ferrera D, et al. (2002): The nuclear protein HMGB1 is secreted by monocytes via a non-classical, vesicle-mediated secretory pathway. EMBO Rep 3: 995-1001.

30. Andersson U, Wang H, Palmblad K, et al. (2000): High mobility group 1 protein (HMG-1) stimulates proinflammatory cytokine synthesis in human monocytes. J Exp Med 192: 565-570. 